\title{
On the Operation of CMOS Active-Cascode Gain Stage
}

\section{Yun Chiu}

Analog and Mixed-Signal Lab, Texas Analog Center of Excellence, University of Texas at Dallas, Richardson, Texas, USA.

Email: chiu.yun@utdallas.edu

Received October 2013

\begin{abstract}
An s-domain analysis of the full dynamics of the pole-zero pair (frequency doublet) associated with the broadly used CMOS active-cascode gain-enhancement technique is presented. Quantitative results show that three scenarios can arise for the settling behavior of a closed-loop active-cascode operational amplifier depending on the relative locations of the unity-gain frequencies of the auxiliary and the main amplifiers. The analysis also reveals that, although theoretically possible, it is practically difficult to achieve an exact pole-zero cancellation. The analytical results presented here provide theoretical guidelines to the design of CMOS operational amplifiers using this technique.
\end{abstract}

Keywords: CMOS Operational Amplifier; Gain Enhancement; Active Cascode; Regulated Cascode; Gain Boosting; Pole- Zero Pair; Doublet; Slow Settling

\section{Introduction}

Invented in 1979 [1] and subsequently refined in 1990 [2-4], the CMOS active-cascode gain-enhancement technique $^{1}$ finds wide applications in analog integrated circuits, such as Nyquist-rate and oversampling data converters, sample-and-hold amplifiers, switched-capacitor filters, band-gap reference circuits, and voltage regulators. By boosting the low-frequency transconductance of the cascode device, the technique increases the output resistance of a CMOS cascode operational amplifier (op amp), and hence the voltage gain without degrading its high-frequency performance. As a result, it is ideally suitable for on-chip applications, where a large gainbandwidth product is desirable while driving capacitive loads. In addition, as the technique derives extra gain laterally using an auxiliary amplifier (booster) without stacking multiple cascode transistors, it retains the highswing feature of a simple cascode stage, and thus, becomes widely popular in scaled CMOS technologies with low supply voltages.

In sampled-data applications, the circuit accuracy and speed are usually determined by the settling behavior of op amps when employed. In an attempt to achieve a high unity-gain frequency and a high dc gain simultaneously, the active cascode introduces a pole-zero pair (doublet) near the unity-gain frequency of the auxiliary amplifier, which potentially leads to slow-settling behavior of such op amps [2]. A guideline to avoid the deleterious effects

${ }^{1}$ Alternative names are gain boosting, regulated cascode, and activefeedback cascode. of the doublet was also discussed in [2]. However, an accurate, closed-form solution of the doublet does not exist. Lacking theoretical guidance, designers often resort to circuit simulators to verify and to fine-tune their op amps, rendering the design process time consuming and heuristic.

This paper examines the doublet behavior of CMOS active cascodes. Quantitative analysis reveals that three scenarios can arise for the closed-loop settling behavior dependent on the ratio of the unity-gain bandwidth of the booster to that of the main amplifier. Sections 2 and 3 review the principles of the cascode gain stage and the CMOS active-cascode technique, respectively. Section 4 presents a small-signal analysis of the active cascode and a closed-form solution of the doublet followed by the corresponding result on settling behavior. In Section 5, computer simulation results are shown to validate the developed theory; and lastly, a brief summary concludes the paper in Section 6.

\section{CMOS Cascode Gain Stage}

Cascode provides a gain-enhancement function in amplifier circuits, allowing the product of the intrinsic gains of two stages-a common-source stage (CS) and a common-gate stage (CG)-to be developed in one. This has an advantage in the attainable bandwidth of the amplifier when driving a capacitive load, which itself acts as the compensation capacitor $[5,6]$. As a result, a single-stage cascode amplifier typically exhibits a better power efficiency relative to a Miller-compensated two-stage design, 
and is widely used in analog circuits.

\subsection{Small-Signal DC Gain}

A typical CMOS cascode gain stage is shown in Figure 1(a) along with its output impedance as a function of frequency in Figure 1(b). In a voltage-gain amplifier, a two-port formulation readily shows that the small-signal gain is simply the product of the effective input transconductance $\left(G_{m}\right)$ and the output resistance $\left(R_{o}\right)$ of the stage [7]. In Fig. 1a, assuming both transistors are biased in saturation, the drain current of $M_{1}$ is only weakly influenced by $M_{2}$ (through channel-length modulation) and the following expression of $G_{m}$ holds:

$$
G_{m}=g_{m 1} \cdot \frac{\left(g_{m 2} r_{o 2}+1\right) r_{o 1}}{\left(g_{m 2} r_{o 2}+1\right) r_{o 1}+r_{o 2}} \approx g_{m 1} .
$$

Thus, the extra gain developed by the cascode can only be explained by the increase in $R_{o}$ :

$$
R_{o}=\left(g_{m 2} r_{o 1}+1\right) r_{o 2}+r_{o 1} \text {. }
$$

Equation (2) is obvious when we consider the stage as a degenerated current source from the output-resistance standpoint, i.e., transistor $M_{2}$ is degenerated by $r_{o 1}$ when the gates of $M_{1}$ and $M_{2}$ are both ac-grounded. The factor $g_{m 2} \cdot r_{o 1}$ is just the loop-gain of the local series feedback formed by $M_{2}$ and $r_{o 1}$. Thus, the dc gain of the stage is

$$
A_{d c}=-G_{m} \cdot R_{o}=-g_{m 1} r_{o 1} \cdot\left(g_{m 2} r_{o 2}+1\right) .
$$

\subsection{Frequency Response}

Next we consider the frequency response of the stage by adding a load capacitor $C_{o}$ to the output. We will neglect all other capacitance in the circuit for simplicity. Since the addition of $C_{o}$ has no effect on the $G_{m}$ part of (1), the frequency dependence derives solely from the output impedance $Z_{o}(s)$, which can be expressed as

$$
Z_{o}(s)=R_{o} \|\left(s C_{o}\right)^{-1}=\frac{R_{o}}{1+s R_{o} C_{o}},
$$

and depicted in Figure 1(b) by the solid curve. Since in this case the $G_{m}$ part exhibits no frequency dependence, the overall frequency response of the small-signal gain is simply

$$
A(s)=-G_{m} \cdot Z_{o}(s)=-\frac{G_{m} R_{o}}{1+s R_{o} C_{o}},
$$

which has a dominant pole at $\omega_{o}\left(\approx 1 / R_{o} C_{o}\right)$ and a unitygain frequency of $\omega_{u}\left(\approx g_{m 1} / C_{o}\right)$.

\section{CMOS Active-Cascode Gain Technique}

CMOS active cascode further improves the achievable dc gain by employing a lateral auxiliary amplifier $-A_{a}(s)$ in Figure 2(a) - to enhance the cascode effect. The opera-

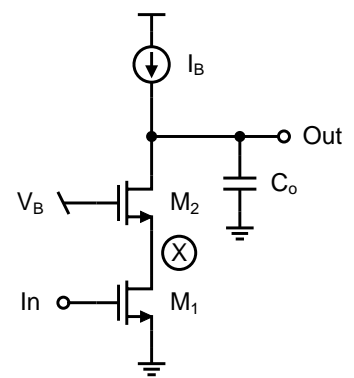

(a)

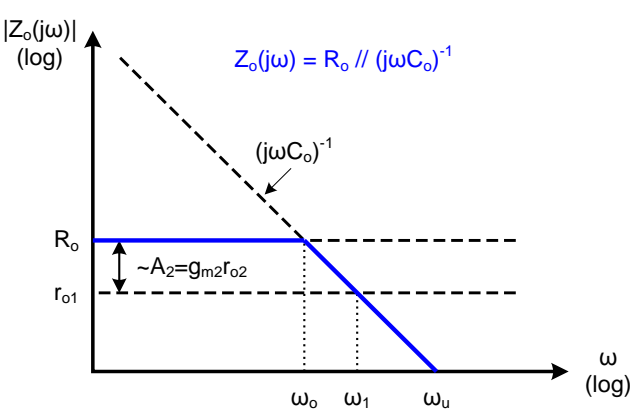

(b)

Figure 1. CMOS cascode gain stage: (a) simplified circuit diagram and (b) Bode plot of the output impedance. The frequency dependence of the output impedance derives from $C_{o}$.

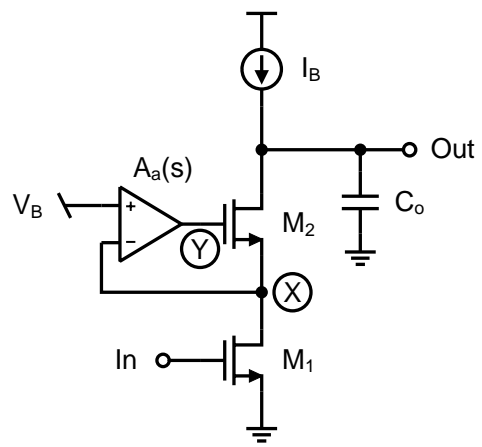

(a)

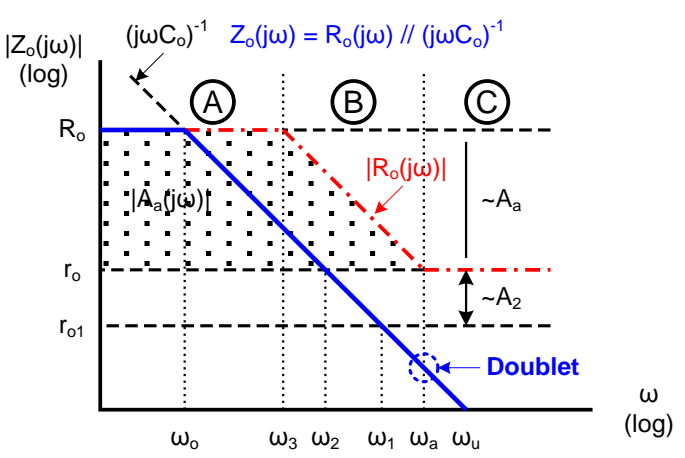

(b)

Figure 2. CMOS active-cascode gain stage: (a) simplified circuit diagram and (b) Bode plot of the output impedance. The frequency dependence of the output impedance derives from $C_{o}$ and the auxiliary amplifier $A_{a}(s)$. 
tion principles of the technique can be explained as follows.

\subsection{Output Resistance and Gain}

At this point, we understand that the extra voltage gain of the normal cascode stage in Figure 1(a) derives from the improved output resistance due to the local series feedback formed by $M_{2}$ and $r_{o 1}$. Therefore, additional gain can be potentially obtained by further increasing either $g_{m 2}$ or $r_{o 1}$. The active-cascode technique exploits the $g_{m 2}$ option as shown in Figure 2(a). Let's consider the effective transconductance of $M_{2}$ due to the presence of the auxiliary amplifier. The gate-source voltage of $M_{2}$ is $0-$ $v_{x}=-v_{x}$ and $v_{y}-v_{x}=-\left(A_{a}+1\right) v_{x}$ before and after the booster insertion, respectively. Therefore, the net effect of the booster is essentially to make the effective transconductance of $M_{2}\left(A_{a}+1\right)$ times larger, with everything else being equal between Figures 1(a) and 2(a). Thus, the dc solution to the active-cascode amplifier of Figure 2(a) can be readily obtained by substituting $\left(A_{a}+1\right) g_{m 2}$ for $g_{m 2}$ in (1)-(3):

$$
\begin{aligned}
G_{m} & =g_{m 1} \cdot \frac{\left[\left(A_{a}+1\right) g_{m 2} r_{o 2}+1\right] r_{o 1}}{\left[\left(A_{a}+1\right) g_{m 2} r_{o 2}+1\right] r_{o 1}+r_{o 2}} \approx g_{m 1}, \\
R_{o} & =\left[\left(A_{a}+1\right) g_{m 2} r_{o 1}+1\right] r_{o 2}+r_{o 1}, \\
A_{d c} & =-g_{m 1} r_{o 1} \cdot\left[\left(A_{a}+1\right) g_{m 2} r_{o 2}+1\right] .
\end{aligned}
$$

We see that the key function of the booster is to enhance $g_{m 2}$, hence to further increase the output resistance (and gain) of the amplifier. ${ }^{3}$ This is done by introducing a push-pull operation between the source and gate voltages of $M_{2}$, i.e., between nodes $X$ and $Y$, through the insertion of a booster amplifier. This inevitably introduces another negative feedback loop that is local to the cascode $M_{2}$. As the bandwidth of the booster amplifier is finite, the frequency response of the active cascode exhibits an interesting artifact-a pole-zero pair or frequency doublet, which will be explained next.

\subsection{Frequency Doublet}

For simplicity, we will assume a single-pole roll-off for the auxiliary amplifier:

$$
A_{a}(s)=\frac{A_{a}}{1+s \frac{A_{a}}{\omega_{a}}} .
$$

${ }^{2}$ The body effect of $M_{2}$ can be readily included in (6)-(8). For example, the dc gain is $A_{d c}=-g_{m 1} r_{o 1} \cdot\left\{\left[\left(A_{a}+1\right) g_{m 2}+g_{m b 2}\right] r_{o 2}+1\right\}$ with body effect.

${ }^{3} \mathrm{~A}$ similar argument also suggests that the technique works only for MOSFET, not BJT amplifiers, in that the base resistance of BJT will ultimately limit the achievable amplifier output resistance to approximately $\beta_{0} r_{o}$, where $\beta_{0}$ is the small-signal current gain of the BJT, regardless of the value of $A_{a}$.
Therefore, the frequency dependence of the gain stage can be readily obtained by replacing $A_{a}$ with $A_{a}(s)$ in (6)-(8).

The effect of $A_{a}(s)$ on the output resistance was qualitatively analyzed in [2], which is sketched in Figure 2(b). Let's examine $R_{o}$ first. The roll-off of $A_{a}(s)$ at high frequency introduces frequency dependence of $R_{o}$ as illustrated by the dash-dotted curve in Figure 2(b), mathematically,

$$
R_{o}(s)=\left[A_{a}(s)+1\right] g_{m 2} r_{o 1} r_{o 2}+r_{o 1}+r_{o 2} .
$$

In Figure 2(b), $r_{o}=g_{m 2} r_{o 1} r_{o 2}+r_{o 1}+r_{o 2}$, which can also be obtained from (10) by setting $A_{a}(s)$ to 0 . Thus, $R_{o}(s)$ exhibits a pole at $\omega_{3}$ and a zero at $\omega_{a}$ in Figure 2(b). The overall output impedance of the stage $Z_{o}(s)$ is then the parallel combination of $R_{o}(s)$ and $\left(s C_{o}\right)^{-1}$,

$$
Z_{o}(s)=R_{o}(s) \|\left(s C_{o}\right)^{-1} \text {, }
$$

which is represented by the solid curve in Figure 2(b).

An equivalent RC model of $Z_{o}(s)$ was proposed in [8], which is sketched in Figure 3. If we divide the frequency axis into three bands by $\omega_{3}$ and $\omega_{a}$ in Figure 2(b), the left resistor $A_{a} r_{o}$ in Figure 3 captures the low-frequency output resistance in region $\mathrm{A}\left(\omega \leq \omega_{3}\right)$, the middle series RC network captures the roll-off part of $R_{o}(s)$ in region B $\left(\omega_{3} \leq \omega \leq \omega_{a}\right)$ and the flat part in region $\mathrm{C}\left(\omega \geq \omega_{a}\right)$, and lastly the right $C_{o}$ represents the shunt load capacitor. In regions $\mathrm{A}$ and $\mathrm{B}$, an approximate expression of the output impedance is

$$
Z_{o}(s) \approx A_{a} r_{o}\left\|\frac{1}{s C_{o}}\right\| \frac{x}{s C_{o}},
$$

where, $x=\omega_{3} / \omega_{o}=\omega_{a} / \omega_{2}$. Obviously, this only results in one pole at $\omega_{0}$. In regions $\mathrm{B}$ and $\mathrm{C}$, an approximate expression of the output impedance is given by

$$
Z_{o}(s) \approx\left(r_{o}+\frac{x}{s C_{o}}\right) \| \frac{1}{s C_{o}}=\frac{1+s \frac{r_{o} C_{o}}{x}}{s C_{o}\left(1+\frac{1}{x}+s \frac{r_{o} C_{o}}{x}\right)} .
$$

Apparently, this results in two poles and one zero, with a pole at $s_{p}=-(1+x) / r_{o} C_{o}$ and a zero at $s_{z}=-x / r_{o} C_{o}$ - both are very close to the unity-gain frequency of the booster $\omega_{a}$ when $x>>1$ holds. This is the pole-zero pair or doublet.

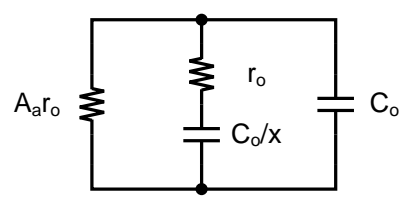

Figure 3. Model of the output impedance of the active cascode in Figure 2. 


\subsection{Slow Settling}

In [9-11], a closed-loop amplifier containing a closely spaced pole-zero pair in its frequency response was examined. It was found that, although the doublet effect on the open-loop Bode plot is often negligible, its deleterious impact on the time-domain settling behavior may be significant. Specifically, it introduces a so-called slow- settling component to the step response of the amplifier. The magnitude of the slow component is proportional to the doublet spacing and the time constant corresponds to the doublet frequency.

The same line of development will be followed here to establish a framework for the next two sections. Let the open-loop transfer function have a closely spaced polezero pair at $\left(\omega_{z}, \omega_{p}\right)$. The dominant pole and unity-gain frequencies, $\omega_{o}$ and $\omega_{u}$, respectively, are given for the open-loop response. When the loop is closed, the feedback will reduce the pole-zero spacing by an amount equal to the loop-gain at the doublet frequency; and the closed-loop pole frequency will move to $\omega_{p}{ }^{\prime}[10,11]$. With the assumption $\omega_{o}<<\left(\omega_{z}, \omega_{p}\right)<<\beta \omega_{u}$, the step response of the closed-loop amplifier is given as

$$
V_{o}(t) \approx V\left(1-k_{1} e^{-\beta \omega_{u} t}+k_{2} e^{-\omega_{p}^{\prime} t}\right),
$$

where, $\beta$ is the feedback factor, and

$$
\omega_{p}^{\prime} \approx \omega_{z}\left(1+\frac{\omega_{z}-\omega_{p}}{\beta \omega_{u}-\omega_{z}}\right) \approx \omega_{z}, \quad k_{2} \approx \frac{\omega_{z}-\omega_{p}}{\beta \omega_{u}} .
$$

The $k_{2}$ term in (14) is the slow-settling component when $\omega_{p}{ }^{\prime}<\beta \omega_{u}$ holds.

\section{Solving Doublet}

For the CMOS active cascode, although the approximate equivalent circuit model of $Z_{o}(s)$ in Section 3.2 reveals the existence of a pole-zero pair near the unity-gain frequency of the auxiliary amplifier $\omega_{a}$, the results are only qualitative as the frequency dependence of $G_{m}(s)$ is not considered in the model. In this section, we will attempt to obtain an exact small-signal solution for the doublet. In the treatment, the frequency dependence of the circuit is assumed to derive from the load capacitor $C_{o}$ and the booster $A_{a}(s)$; and all other capacitances will be neglected first to keep the math tractable. After the first-order polezero behavior is derived, the effects of other capacitances in the circuit will be examined in Section 5 using computer simulation.

\subsection{Open-Loop Transfer Function}

To solve for the open-loop transfer function, we first obtain the expressions for $G_{m}(s)$ and $Z_{o}(s)$ :

$$
\begin{gathered}
G_{m}(s)=g_{m 1} \cdot \frac{\left[\left(A_{a}(s)+1\right) g_{m 2} r_{o 2}+1\right] r_{o 1}}{\left[\left(A_{a}(s)+1\right) g_{m 2} r_{o 2}+1\right] r_{o 1}+r_{o 2}}, \\
Z_{o}(s)=R_{o}(s) \| \frac{1}{s C_{o}}=\frac{R_{o}(s)}{1+s C_{o} R_{o}(s)} .
\end{gathered}
$$

The product of (16) and (17) gives the small-signal voltage gain in (18) (see below), where $A_{1}=g_{m 1} \cdot r_{o 1}, A_{2}=$ $g_{m 2} \cdot r_{o 2}$, and the expression of $A_{\mathrm{a}}(s)$ in (9) is assumed.

\subsection{Frequency Doublet}

The numerator of (18) readily solves to one LHP zero:

$$
S_{z}=-\left(\frac{A_{2}}{A_{2}+1}+\frac{1}{A_{a}}\right) \omega_{a} \approx-\omega_{a} .
$$

To solve for the poles, we define $\gamma=r_{o 2} r_{o 1}, \omega_{u}=$ $g_{m 1 /} C_{o}$, and $r_{o 1}=A_{1 /} C_{o} \omega_{u}$; the denominator of (18) reduces to the following:

$$
\begin{gathered}
s^{2}+\left[\frac{\left(A_{a}+1\right) A_{2}+1+\gamma}{A_{a}\left(A_{2}+1+\gamma\right)} \omega_{a}+\frac{\omega_{u}}{A_{1}\left(A_{2}+1+\gamma\right)}\right] s \\
+\frac{\omega_{u} \omega_{a}}{A_{a} A_{1}\left(A_{2}+1+\gamma\right)}=0 .
\end{gathered}
$$

Due to the presence of the doublet, we may assume that one pole is at $s_{p 1}=-\alpha \omega_{a}$ with $\alpha \approx 1$; and (20) can be factorized into

$$
\left(s+\alpha \omega_{a}\right) \cdot\left(s+\frac{\omega_{u}}{\alpha A_{a} A_{1}\left(A_{2}+1+\gamma\right)}\right)=0 .
$$

Compare (21) with (20), we obtain

$$
\alpha \approx \frac{A_{2}}{A_{2}+1+\gamma}+\frac{1}{A_{a}}+\frac{A_{a}-1}{A_{a} A_{1}\left(A_{2}+1+\gamma\right)} \frac{\omega_{u}}{\omega_{a}},
$$

where, $\frac{\omega_{u}}{A_{a} A_{1}\left(A_{2}+1+\gamma\right)} \approx \omega_{o}<<\omega_{a}$ is assumed since $x>>$ 1. At this point, we arrive at the solution for the two LHP poles of the open-loop transfer function:

$$
\begin{aligned}
& -S_{p 1} \approx \frac{\omega_{u}}{A_{1}\left[\left(A_{a}+1\right) A_{2}+1+\gamma\right]+\left(A_{a}-1\right) \frac{\omega_{u}}{\omega_{a}}} \approx \omega_{o}, \\
& -S_{p 2} \approx\left(\frac{A_{2}}{A_{2}+1+\gamma}+\frac{1}{A_{a}}\right) \omega_{a}+\frac{A_{a}-1}{A_{a} A_{1}\left(A_{2}+1+\gamma\right)} \omega_{u} \approx \omega_{a},
\end{aligned}
$$


where, the fact $x=\omega_{3 /} \omega_{0}>>1$ is again assumed.

\subsection{Closed-Loop Settling Behavior}

We recognize that $s_{p 1}$ of (23) is the dominant pole of the open-loop amplifier and $\left(s_{z}, s_{p 2}\right)$ of (19) and (24) form a doublet. Substituting (19) and (24) in (15) results in an expression for the slow-settling component:

$$
k_{2} \approx \frac{1}{\beta}\left[\frac{\omega_{p}{ }^{\prime} \approx \omega_{a},}{\left(A_{2}+1\right)\left(A_{2}+1+\gamma\right)} \frac{\omega_{a}}{\omega_{u}}-\frac{A_{a}-1}{A_{a} A_{1}\left(A_{2}+1+\gamma\right)}\right] .
$$

Equation (26) reveals that three scenarios can arise for the closed-loop settling behavior dependent on $\omega_{a /} \omega_{u}$, the ratio of the unity-gain bandwidth of the auxiliary amplifier to that of the open-loop main amplifier:

1) $k_{2}=0$, critically damped, when $\omega_{u} \omega_{a} \approx \gamma A_{1}=g_{m 1} \cdot r_{o 2}$. The pole cancels the zero exactly and the slow-settling term vanishes;

2) $k_{2}>0$, overshoot, when $\omega_{u /} \omega_{a}<g_{m 1} \cdot r_{o 2}$. This results in a falling doublet;

3) $k_{2}<0$, slow settling, when $\omega_{u l} \omega_{a}>g_{m 1} \cdot r_{o 2}$. This results in a rising doublet, the one often cited in the literature [2].

In addition, as $\omega_{u}=g_{m 1} C_{o}$, the criterion $\omega_{u /} \omega_{a}=g_{m 1} \cdot r_{o 2}$ leads to $\omega_{a}=1 / r_{o 2} C_{o}$. In other words, even when a constant $C_{o}$ is assumed, the significant dependence of $r_{o 2}$ on the amplifier output voltage makes it practically difficult to achieve an exact pole-zero cancellation.

There are two strategies to avoid the deleterious effect of the doublet. One suggests to make $\omega_{a}>\beta \omega_{u}$ to avoid slow settling [2]. The other suggests to make $k_{2}$ small enough, i.e., to have a "slow-but-accurate" doublet. Let's examine the feasibility of the latter. Assuming a very slow doublet, i.e., $\omega_{a}<<\beta \omega_{u}$ holds, then following (26),

$$
k_{2} \approx-\frac{1}{\beta A_{1} A_{2}} \approx-\frac{1}{\beta A_{0}} .
$$

This implies that $k_{2}$ cannot be made arbitrarily smallthe smallest value of $k_{2}$ is inversely proportional to the loop-gain of the main amplifier without gain enhancement. A "slow-but-accurate" doublet does not exist.

\section{Computer Simulation}

Computer simulations have been performed to validate the analysis developed in Section 4. For easy access to and programmability of all device parameters, i.e., $g_{m}, r_{o}$, etc., a small-signal linear model of the active cascode shown in Figure 4 was used instead of a real transistor circuit. An ideal VCVS models the auxiliary amplifier with a transfer function $A_{a}(s)$. In Figure 5, the external feedback is assumed ideal with a feedback factor $\beta=1 / 2$ (i.e., the closed-loop gain is 2); and a 1-V step is applied at the input. A capacitor $C_{m}$ on node $X$ (resulting in the

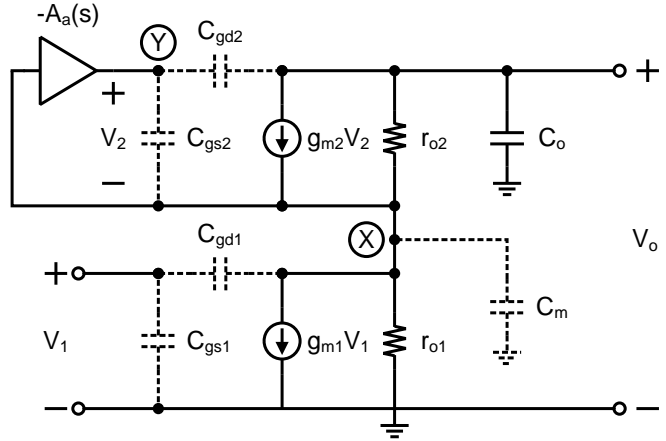

Figure 4. Small-signal model of the CMOS active cascode. A capacitor $C_{m}$ on node $X$ and the $C_{g s}$ ' and $C_{g d}$ 's of $M_{1}$ and $M_{2}$ are also included for second-order effects.

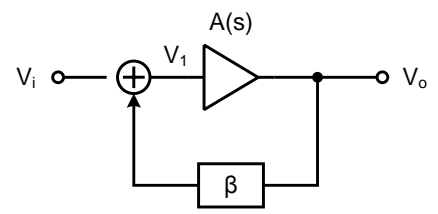

(a)

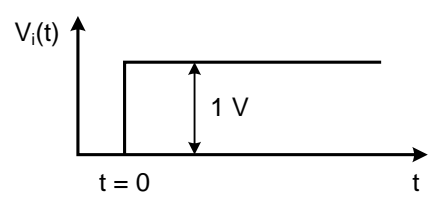

(b)

Figure 5. (a) Closed-loop amplifier model and (b) the input voltage step used in simulation.

second pole) and the $C_{g s}$ ' and $C_{g d}$ 's of $M_{1}$ and $M_{2}$ are also included for completeness. All device parameters used in the simulation are listed in Table $\mathbf{1}$.

\subsection{Intrinsic Doublet Behavior}

To evaluate the intrinsic behavior of the doublet, all capacitors are removed except $C_{o}$ in Figure 4; the unity-gain frequency of $A_{a}(s)$ is swept to observe the settling behavior of the closed-loop amplifier. Figures 6(a) and (b) show the output voltage and the normalized settling error of the circuit, respectively. The settling error is defined as

$$
\delta(t)=\frac{V_{o}(t)-\frac{V_{i}(t)}{\beta}}{\frac{V_{i}(t)}{\beta}},
$$

It is apparent that indeed three scenarios for the settling behavior exist as predicted in Section 4.3. Specifically, a critically damped output transient was observed confirming the possibility of an exact pole-zero cancellation. In the example used here, (26) predicts that $k_{2}=0$ when $f_{a}=\left(2 \pi r_{o 2} C_{o}\right)^{-1} \approx 10 \mathrm{MHz}$; while computer simula- 
Table 1. Small-signal parameters used in simulation.

\begin{tabular}{cccc}
\hline$\beta$ & 0.5 & $r_{o 2}$ & $10 \mathrm{k} \Omega$ \\
$f_{u}$ & $200 \mathrm{MHz}$ & $C_{m}$ & $C_{o} / 3$ \\
$A_{a}$ & $40 \mathrm{~dB}$ & $C_{g s 1}$ & $C_{o} / 6$ \\
$g_{m 1}$ & $2 \mathrm{~mA} / \mathrm{V}$ & $C_{g s 2}$ & $C_{o} / 6$ \\
$g_{m 2}$ & $1 \mathrm{~mA} / \mathrm{V}$ & $C_{g d 1}$ & $C_{o} / 12$ \\
$r_{o 1}$ & $10 \mathrm{k} \Omega$ & $C_{g d 2}$ & $C_{o} / 12$ \\
\hline
\end{tabular}

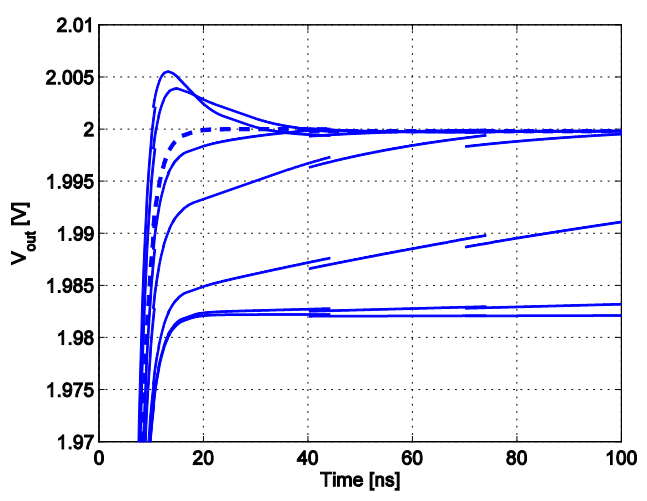

(a)

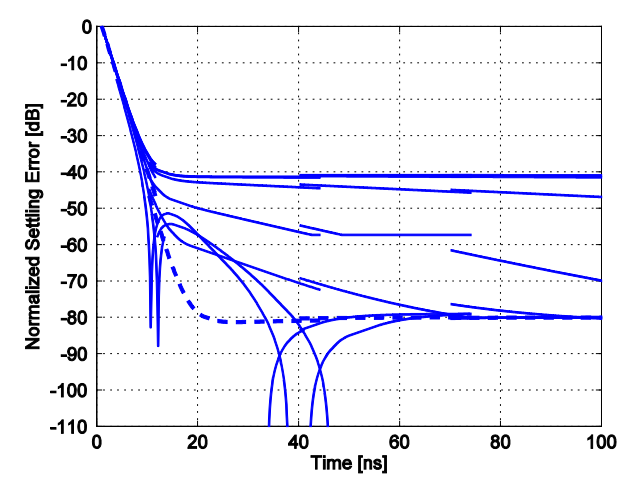

(b)

Figure 6. Closed-loop settling behavior for $f_{a}=10 \mathrm{kHz}, 100$ kHz, 1 MHz, 5 MHz, 9 MHz, 10.95 MHz, 20 MHz, and 30 MHz: (a) the amplifier output voltage (increasing in $f_{a}$ from bottom up), and (b) the normalized settling error (increasing in $f_{a}$ from top down). Open-loop parameters: $f_{a}=10$ $\mathrm{kHz}, \beta f_{u}=100 \mathrm{MHz}$, and $\beta A_{d c}=80 \mathrm{~dB}$. The dashed curves $\left(f_{a}=10.95 \mathrm{MHz}\right)$ correspond to the case of $k_{2}=0$, i.e., exact pole-zero cancellation.

tion reveals that this actually occurs for $f_{a} \approx 10.95 \mathrm{MHz}$. In addition, Figure 6(b) further indicates that, when the slow-settling component is really "slow" $\left(\omega_{a}<<\beta \omega_{u}\right)$, the knee accuracy where the slow term starts to dominate is always nearly $-40 \mathrm{~dB}$, corresponding to $k_{2}=1 / \beta A_{o}=$ -0.01 as predicted by (27).

\subsection{Effect of the Second Pole}

A capacitor $C_{m}$ at node $X$ in Figure 4 is added to intro- duce a non-dominant pole with a frequency three times larger than $\beta \omega_{u}$, the close-loop bandwidth (i.e., the phase margin of the loop-gain is around $71^{\circ}$ ). The simulation results are shown in Figures 7(a) and (b). The basic settling behavior is unaltered with the inclusion of the second pole; and the slow tails after the initial overshoots die out closely resemble those of the case with $C_{m}=0$, especially when $\omega_{a}<<\beta \omega_{u}$ holds.

\subsection{Effect of Other Parasitics}

The $C_{g s}$ ' and $C_{g d}$ 's of the transistors $M_{1}$ and $M_{2}$ are further included to make the small-signal model complete. Reasonably large values for these capacitors are assumed (Table 1), and the simulation results are shown in Figures 8(a) and (b). Again, the behavior of the slow-settling component is seemingly independent of the various second-order effects introduced by the parasitics. An exact pole-zero cancellation always occurs for $f_{a} \approx 10.95$ $\mathrm{MHz}$.

In [2], the criterion $\beta \omega_{u}<\omega_{a}<\omega_{p 2}$ was proposed to ensure proper operation of the active cascodes. This is verified in simulation by keeping $C_{m}$ constant while stepping $\omega_{a}$ up to and beyond $\omega_{p 2}$. Figure 9 illustrates

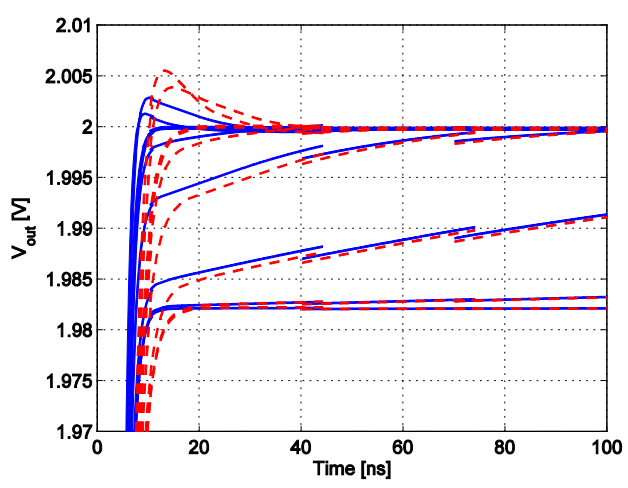

(a)

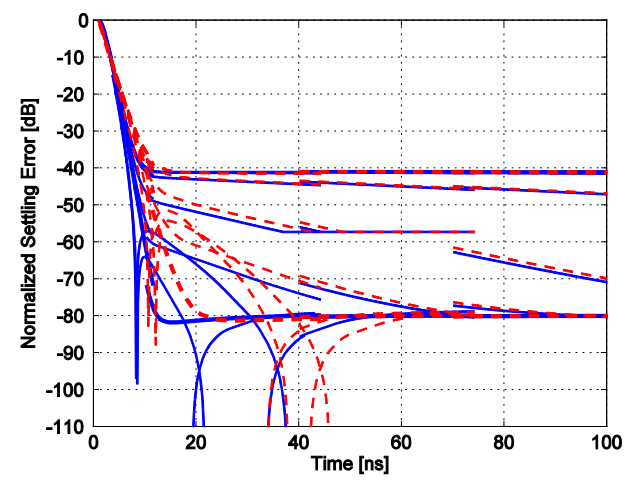

(b)

Figure 7. The same results as those of Figure 6 with the inclusion of $C_{m}$. The phase margin of the loop-gain is $71^{\circ}$. The dashed curves are for $C_{m}=0$. The basic settling behavior remains the same in the presence of a second pole. 


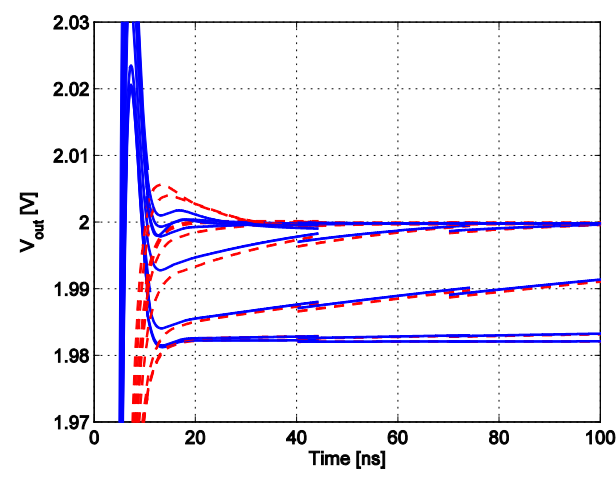

(a)

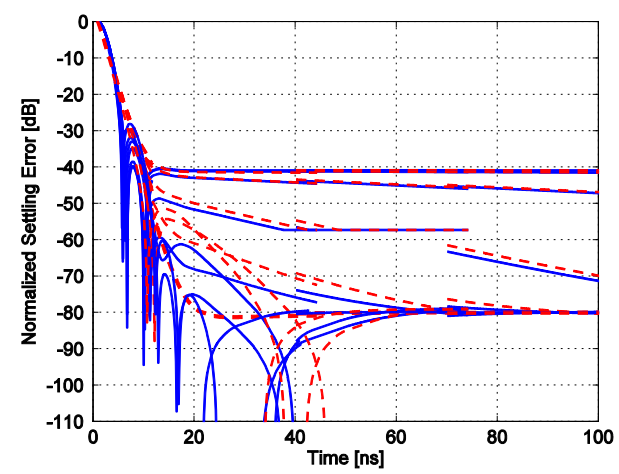

(b)

Figure 8. The same results as those of Figure 6 with the inclusion of $C_{m}$ and the $C_{g s}$ ' and $C_{g d}$ 's of $M_{1}$ and $M_{2}$. The slow tails of the transients closely resemble those of the original case (the dashed curves) in spite of the initial significant overshoots.

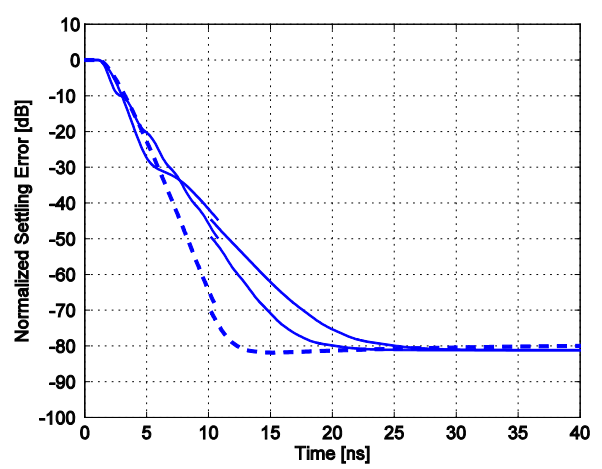

Figure 9. The normalized settling error for $f_{a}=10.95 \mathrm{MHz}$ (dashed curve), $100 \mathrm{MHz}$, and $1 \mathrm{GHz}$ with $f_{p 2} \approx 300 \mathrm{MHz}$.

that sluggish settling occurs when $\omega_{a}$ is in the vicinity of $\omega_{p 2}$, where the local feedback loop formed by the booster and the cascode becomes marginally stable. The instability is lifted when $\omega_{a}$ is further pushed out (not shown in the figure).

\section{Summary}

An accurate, closed-form $s$-domain analysis and comput- er simulation results of the pole-zero pair (doublet) associated with the widely used CMOS active-cascode gain stage are presented. The conventional picture of "slow settling" is clarified and augmented with a set of equations that completely describe the doublet dynamics. These results provide easy-to-follow guidelines to the design of such amplifiers in practice.

\section{Acknowledgements}

This work was inspired by a conversation took place between the author and Klaas Bult at UCLA in 1996. The small-signal solution of the doublet was developed in 1997.

\section{REFERENCES}

[1] B. J. Hosticka, "Improvement of the Gain of MOS Amplifiers,” IEEE Journal of Solid-State Circuits, Vol. 14, No. 6, 1979, pp. 1111-1114. http://dx.doi.org/10.1109/JSSC.1979.1051324

[2] K. Bult and G. J. G. M. Geelen, “A Fast-Settling CMOS Op Amp for SC Circuits with 90-dB DC Gain,” IEEE Journal of Solid-State Circuits, Vol. 25, No. 6, 1990, pp. 1379-1384. http://dx.doi.org/10.1109/4.62165

[3] E. Sackinger and W. Guggenbuhl, "A High-Swing, High-Impedance MOS Cascode Circuit,” IEEE Journal of Solid-State Circuits, Vol. 25, No. 1, 1990, pp. 289-298. http://dx.doi.org/10.1109/4.50316

[4] H. C. Yang and D. J. Allstot, “An Active-Feedback Cascode Current Source,” IEEE Transactions on Circuits and Systems, Vol. 37, No. 5, 1990, pp. 644-646. http://dx.doi.org/10.1109/31.55008

[5] P. R. Gray and R. G. Meyer, "MOS Operational Amplifier design-A Tutorial Overview," IEEE Journal of Solid-State Circuits, Vol. 17, No. 6, 1982, pp. 969-982. http://dx.doi.org/10.1109/JSSC.1982.1051851

[6] A. A. Abidi, "On the Operation of Cascode Gain Stages," IEEE Journal of Solid-State Circuits, Vol. 23, No. 6, 1988, pp. 1434-1437. http://dx.doi.org/10.1109/4.90043

[7] P. R. Gray, P. J. Hurst, S. H. Lewis and R. G. Meyer, "Analysis and Design of Analog Integrated Circuits," 4th Edition, Wiley, New York, 2001.

[8] K. Bult, “EE215A Course Notes,” UCLA, 1996.

[9] F. D. Waldhauer, "Analog Integrated Circuits of Large Bandwidth,” IEEE International Convention Record, Vol. 11, Part 2, 1963, pp. 200-207.

[10] P. R. Gray and R. G. Meyer, "Recent Advances in Monolithic Operational Amplifier Design,” IEEE Transactions on Circuits and Systems, Vol. 21, No. 3, 1974, pp. 317-327. http://dx.doi.org/10.1109/TCS.1974.1083851

[11] B. Y. T. Kamath, R. G. Meyer and P. R. Gray, "Relationship between Frequency Response and Settling Time of Operational Amplifiers," IEEE Journal of Solid-State Circuits, Vol. 9, No. 6, 1974, pp. 347-352. http://dx.doi.org/10.1109/JSSC.1974.1050527 